\title{
Peptic ulcer disease
}

A peptic ulcer, also known as peptic ulcer disease (PUD) is the most common ulcer of an area of the gastrointestinal tract that is usually acidic and thus extremely painful. It is defined as mucosal erosions equal to or greater than $0.5 \mathrm{~cm}$. As many as $70 \%-90 \%$ of such ulcers are associated with Helicobacter pylori, a spiral-shaped bacterium that lives in the acidic environment of the stomach.

\section{History}

John Lykoudis, a general practitioner in Greece, treated patients for peptic ulcer disease with antibiotics, beginning in 1958, long before it was commonly recognized that bacteria were a dominant cause for the disease.

Helicobacter pylori was rediscovered in 1982 by two Australian scientists, Robin Warren and Barry J. Marshall as a causative factor for ulcers. In their original paper, Warren and Marshall contended that most gastric ulcers and gastritis were caused by colonization with this bacterium, not by stress or spicy food as had been assumed before.

In 2005, the Karolinska Institute in Stockholm awarded the Nobel Prize in Physiology or Medicine to Dr. Marshall and his long-time collaborator Dr. Warren "for their discovery of the bacterium Helicobacter pylori and its role in gastritis and peptic ulcer disease." Professor Marshall continues research related to $H$. pylori and runs a molecular biology lab at UWAin Perth, Western Australia.

\section{Epidemiology}

Peptic ulcer disease had a tremendous effect on morbidity and mortality until the last decades of the 20th century, when epidemiological trends started to point to an impressive fall in its incidence. The reason that the rates of peptic ulcer disease decreased is thought to be the development of new effective medication and acid suppressants and the discovery of the cause of the condition, H. pylori.

\section{Classification}

\section{Modified Johnson Classification of peptic ulcers:}

Type I: Ulcer along the body of the stomach, most often along the lesser curve at incisura angularis along the locus minoris resistantiae.

Type II: Ulcer in the body in combination with duodenal ulcers. Associated with acid over secretion.

Type III: In the pyloric channel within $\mathrm{cm}$ of pylorus. Associated with acid over secretion.

Type IV: Proximal gastro esophageal ulcer.

Type V: Can occur throughout the epigastric region Associated with chronic NSAID use (such as aspirin).

\section{Signs and symptoms}

\section{What Are the Symptoms of an Ulcer?}

An ulcer may or may not have symptoms. When symptoms occur, they may include:

A burning pain in the middle or upper stomach between meals or at night

Bloating

Heartburn

Nausea or vomiting

In severe cases, symptoms can include:

Dark or black stool (due to bleeding)

Vomiting blood (that can look like "coffee-grounds")

Weight loss

Severe pain in the mid to upper abdomen

\section{Causes}

A major causative factor ( $60 \%$ of gastric and up to $90 \%$ of duodenal ulcers) is chronic inflammation due to Helicobacter pylori that colonizes the antral mucosa. The immune system is unable to clear the infection, despite the appearance of antibodies. Thus, the bacterium can cause a chronic active gastritis (type B gastritis), resulting in a defect in the regulation 
of gastrin production by that part of the stomach.Gastrin stimulates the production of gastric acid by parietal cells. In H. pylori colonization responses to increased gastrin, the increase in acid can contribute to the erosion of the mucosa and therefore ulcer formation.

\section{Predisposing factors: Diet}

The incidence of peptic ulcers was higher with staple diets of refined wheat flour and polished rice and much lower where the staple diets were village ground millet and pulses. A mix of Phospholipids and phytosterol in these diets will prevent ulcers where rats are exposed to ulcerogenic treatment with NSAIDS. $H$ Pylori has a similar incidence in both populations. The combination of phospholipids and phytosterols may be of value in the prevention and treatment of duodenal ulceration and protection against the ulcerogenic effect of NSAIDs.

\section{Diagnosis}

The diagnosis is mainly established based on the characteristic symptoms. Confirmation of the diagnosis is made with the help of tests such as endoscopies or barium contrast $\mathrm{x}$-rays. The tests are typically ordered if the symptoms do not resolve after a few weeks of treatment, or when they first appear in a person who is over age 45 or who has other symptoms such as weight loss, because stomach cancer can cause similar symptoms.

One of the reasons that blood tests are not reliable for accurate peptic ulcer diagnosis on their own is their inability to differentiate between past exposure to the bacteria and current infection.

The diagnosis of Helicobacter pylori can be made by:

I) Urea breath test (noninvasive and does not require EGD,Esophagogastroduodenostomy)

II) Direct culture from an EGD biopsy specimen; this is difficult to do, and can be expensive. Most labs are not set up to perform $\mathrm{H}$. pylori cultures;

III) Direct detection of urease activity in a biopsy specimen by rapid urease test;

IV) Measurement of antibody levels in blood (does not require EGD). It is still somewhat controversial whether a positive antibody without EGD is enough to warrant eradication therapy;

V) Stool antigen test;

VI) Histological examination and staining of an EGD biopsy.

\section{Who Is More Likely To Get Ulcers?}

You may be more likely to develop ulcers if you:

Are infected with the H. pylori bacterium

Take NSAIDs such as aspirin, ibuprofen, or naproxen

Have a family history of ulcers

Have another illness such as liver, kidney, or lung disease

Drink alcohol regularly

Are 50 years old or older

\section{Complications of PUD}

Though ulcers often heal on their own, you shouldn't ignore their warning signs. If not properly treated, ulcers can lead to serious health problems, including:

Bleeding

Perforation (a hole through the wall of the stomach)

Gastric outlet obstruction.

Carcinoma stomach.

\section{Treatment}

The conventional treatment of PUD focuses on killing the bacteria and reducing the level of acid in the stomach to relieve pain and encourage healing.

I) An effective first-line therapy for uncomplicated cases would be Amoxicillin + Metronidazole + Pantoprazole (PPI).

II) When H. pylori infection is present, the most effective treatments are combinations of 2 antibiotics (e.g. Clarithromycin, Amoxicillin, Tetracycline, Metronidazole) and 1 proton pump inhibitor (PPI), sometimes together with a bismuth compound.

III) In complicated, treatment-resistant cases, 3 antibiotics (e.g. amoxicillin + clarithromycin + metronidazole) may be used together with a PPI and sometimes with bismuth compound.

IV) In the absence of $H$. pylori, long-term higher dose PPIs are often used.

\section{Diet and nutrition in ulcer disease}

In this era of H2-inhibitors, the available evidence does not support the need to place peptic ulcer disease patients on restrictive diets. The major goal of diet is to avoid extreme elevations of gastric acid secretion and the direct irritation of gastric mucosa. Frequent milk singestion as previously prescribed is not encouraged. This is owing to the transient buffering effect and ignificant gastric acid secretion effect of milk. The fat content of milk has no influence on these effects. 
Spices, in particular black pepper, red pepper, and chili powder, may produce dyspepsia. One study shows red chili powder to have no detrimental effect on duodenal ulcer healing. It has also been proposed that daily pepper ingestion may have a beneficial adaptive cytoprotective response. While still controversial and under evaluation, peptic ulcer patients should avoid any spice that causes discomfort, especially during exacerbation of peptic disease. Coffee should be avoided on the basis of its strong acid secretogenic property. Coffee can induce dyspepsia. Whether noncoffee caffeine-containing beverages (tea, soft drinks) induce peptic ulcer is unknown, but they are acid secretion stimulators. There appears to be no evidence to restrict dietary fiber. Some fiber-containing foods may possess factors that are protective against ulcer disease. According to the Mayo Clinic Diet Manual, previously recommended small frequent feedings have not been shown to be more effective than three meals per day in the treatment of chronic peptic ulcer disease. This reference cites authorities advising against extra feedings because of increased acid secretion and unnecessary complication of eating patterns. However, some patients claim to be relieved of symptoms with more frequent feedings, especially during acute phases. Citric acid juices may induce reflux and cause discomfort in selective patients. Stomach distention with large quantities of food should be discouraged.

\section{Peptic Ulcer Diet: Points To Remember}

- Eat 5 to 6 small meals a day instead of 3 larger meals. It is important that you avoid overeating. Frequent, smaller meals will be more comfortable and easier on the stomach than two or three large meals a day.

- Eat a diet rich in fiber, especially from fruits and vegetables

- Rest and relax a few minutes before and after each meal, as well as remaining relaxed during meals.

- Eat slowly and chew the food well

- Avoid eating within 3 hours before bedtime

- Eat foods that are low fat
- Avoid foods that are fried

- Avoid foods that are spicy

\section{Cut down on the following foods}

- Coffee

- Decaffeinated coffee

- Tea

- Cola drinks

- Carbonated beverages

- Citrus fruits

- Tomato-based products

- Chocolate

- Avoid alcohol

- Quit smoking

\section{Mind/Body Medicine}

Make serious efforts to neutralize stress in your life by practicing healthy stress management techniques such as breathing exercises and meditation, learning visualization or hypnotherapy (or both) to heal your ulcer, or by taking a course of biofeedback training. As necessary, create positive changes in whatever in your life causes you the most stress - your job, living situation, or relationships.

\section{Conclusion}

With modern treatment, patients with peptic ulcer disease can lead normal lives without lifestyle changes or dietary restrictions. Cigarette smokers have been found to have more complications from ulcers and treatment failure. Eradication of the bacteria H. pylori not only heals ulcers but also prevents the recurrence of ulcer disease. 